\title{
METODOLOGÍA DE VALORACIÓN PARA PROYECTOS DE TRANSFERENCIA TECNOLÓGICA UNIVERSITARIA. CASO APLICADO - UNIVERSIDAD DE ANTIOQUIA*
}

\author{
JAIME ANDRÉS CORREA GARCÍA**, MARTÍN DARÍO ARANGO SERNA*** \& KARLA CRISTINA ALVAREZ URIBE**** \\ UNIVERSIDAD DE ANTIOQUIA; UNIVERSIDAD NACIONAL DE COLOMBIA \\ - FACULTAD DE MINAS SEDE MEDELLÍN
}

Recibido/ Received/ Recebido: 09/05/2011 - Aceptado/ Accepted/Aprovado: 10/01/2012

\begin{abstract}
Resumen
Este artículo propone lineamientos para iniciar el camino de la definición y estructuración de una metodología para la valoración de los proyectos de transferencia tecnológica universitaria, puesto que la misión actual de las universidades se revalúa y trasciende más allá de la docencia, la investigación y la extensión. Actualmente, se requiere que la Universidad transfiera el conocimiento y los resultados de la acumulación de capital intelectual en beneficio de la sociedad. Igualmente, se desarrolla con relación a la valoración de los proyectos de transferencia tecnológica universitaria una metodología que facilite realizar los esquemas de transferencia existentes y los modelos de valoración del capital intelectual.
\end{abstract}

Palabras clave: capital intelectual, transferencia tecnológica universitaria, grupos de investigación, spin off.

\section{VALUATION METHODOLOGY FOR PROJECTS OF UNIVERSITY TECHNOLOGICAL TRANSFERENCE. CASE OF STUDY - UNIVERSITY OF ANTIOQUIA.}

\begin{abstract}
This article proposes the guidelines to initiate the road of definition and structuration of a valuation methodology for projects of university technological transference. Thus, the current mission of the universities is being revaluated and goes beyond teaching, research and courses of extension. $\mathrm{Cu}$ rrently, the university must to transfer knowledge and the results from intellectual capital accumulation for the benefit of society. Additionally, a methodology to make easier the elaboration of the existent transference schemes and valuation models of intellectual capital are developed, in relation to technological transference projects valuation.
\end{abstract}

Keywords: intellectual capital, university technological transferences, research groups, spin off.

* $\quad$ Este artículo es producto del proyecto de investigación sobre valoración de la trasferencia tecnológica del trabajo final de grado en la Maestría en Ingeniería Administrativa del estudiante Jaime Andrés Correa, coautor de este articulo. Dicho proyecto fue financiado por la Universidad de Antioquia- Departamento de Ciencias Contables en el año 2009. del grupo de investigaciones y consultorías en ciencias contables -GICCO-. Correo electrónico: jaimecorrea@economicas.udea. edu.co

*** $\mathrm{PhD}$. En Ingeniería Industrial. Profesor Titular Universidad Nacional de Colombia - Sede Medellín. Director del Grupo de Investigación I+D+I Logística Industrial- Organizacional -GICO-. Correo electrónico: mdarango@unal.edu.co

**** Estudiante de Maestría en Ingeniería Administrativa. Integrante del Grupo de Investigación I+D+I Logística Industrial- Organizacional -GICO-. Correo electrónico: kcalvare@unal.edu.co 


\title{
METODOLOGIA DE AVALIAÇÃO PARA PROJETOS DE TRANSFERÊNCIA TECNOLÓGICA UNIVERSITÁRIA. O CASO DA UNIVERSIDADE DE ANTIOQUIA
}

\begin{abstract}
Resumo
Este artigo propõe lineamentos para iniciar o caminho da definição e da estruturação de uma metodologia para a avaliação dos projetos de transferência tecnológica universitária. Posto que a avaliação da missão atual das universidades vai para além da docência, da pesquisa e da extensão, atualmente se requer que a Universidade transfira o conhecimento e os resultados da acumulação de capital intelectual em benefício da sociedade. Igualmente, desenvolve-se com relação à avaliação dos projetos de transferência tecnológica universitária uma metodologia que facilite realizar os esquemas de transferência existentes e os modelos de avaliação do capital intelectual.

Palavras chave: capital intelectual, transferência tecnológica universitária, grupos de investigação, spin off.

Correa, J., Arango, M. \& Álvarez, K. (2012) Metodología de valoración para proyectos de transferencia tecnológica universitaria. Caso aplicado - Universidad de Antioquia. En: Revista de la Facultad de Ciencias Económicas de la Universidad Militar Nueva Granada. rev.fac.cienc.econ, XX (1)
\end{abstract}

JEL: M14, M15.

\section{Introducción}

La misión actual de las universidades se revalúa y trasciende más allá de la docencia, la investigación y la extensión. Hoy por hoy, se requiere que la Universidad transfiera el conocimiento y los resultados de la acumulación de capital intelectual en beneficio de la sociedad. En este sentido, se dinamizan las relaciones entre la Universidad, el Estado y la Empresa; con lo cual surgen relaciones y procesos de negociación en los cuales se requiere realizar valoraciones que faciliten la transferencia tecnológica de conocimiento. Para la valoración de los proyectos de transferencia tecnológica universitaria se requiere analizar los esquemas de transferencia existentes y los modelos de valoración del capital intelectual.

El presente artículo se enmarca en las dinámicas de trabajo en las que actualmente se encuentra la Universidad Colombiana y las universidades del denominado G- ${ }^{1}$, donde la generación de nuevos proyectos de alto valor agregado e innovación para dar respuesta a necesidades sociales, es una prioridad y un objetivo definido en los acuerdos que estipulan dichas universidades.

Se propone una metodología que posibilite la valoración de proyectos de transferencia tecnológica por parte de las universidades y en particular por la Universidad de Antioquia. El proyecto de transferencia tecnológica analizado es CONOSER (Conocimiento y servicios de ingeniería) derivado del Grupo de Investigación Manejo Eficiente de la Energía - GIMEL.

\section{Propuesta para la aplicación de la va- loración a los proyectos de transferencia tecnológica universitaria}

En el proceso de la valoración se identifican cuatro componentes determinantes para llevarlo a cabo, los cuales son: Proyecto, grupo de investigación, institución y recursos financieros, tal como se plantean en la Ilustración 1.

\footnotetext{
Universidad de Antioquia, Universidad Nacional de Colombia Sede Medellín, Universidad EAFIT, Universidad de Medellín, Universidad Pontificia Bolivariana, Universidad CES, Escuela de Ingeniería de Antioquia y Corporación Universitaria Lasallista.
} 
Ilustración 1. Rombo de la valoración de TTU²

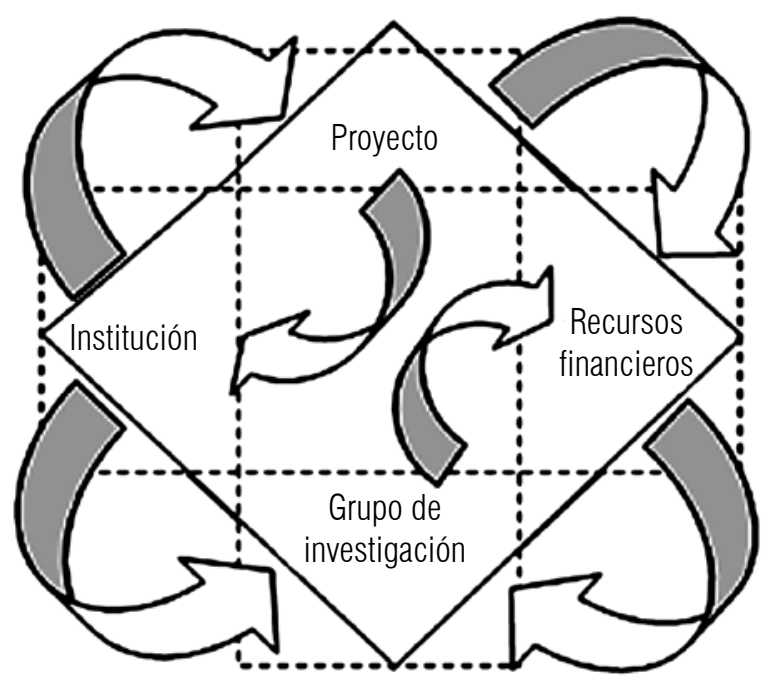

Es posible identificar la interacción de los diferentes componentes que hacen parte de la valoración de Transferencia Tecnológica Universitaria -TTU-. Se describe a continuación cada uno de ellos.

- Proyecto: Comprende aspectos como la innovación y creatividad que se desarrolla en la construcción y proyección del proyecto elaborado por el grupo de investigación y que integra el conocimiento plasmado a través del diseño de un plan para definir un nuevo bien o servicio derivado de un proceso de investigación que surge al interior de una institución. Es clave que esta planeación ${ }^{3}$ sea clara con el fin de realizar una evaluación de la capacidad del proyecto para producir flujos de caja futuros para aquellos interesados en la propuesta, así como, la factibilidad en la ejecución que dependerá de diversos factores tales como: Impactos ambientales, sociales, legislación, mercado interesado, entre otros.

- Institución: El respaldo de la institución en la cual es desarrollado el proyecto es clave en el proceso de investigación, contando con recursos tangibles (Planta física, laboratorios, equipos de cómputo, etc.,) e intangibles (trayectoria, reconocimiento-credibilidad, conocimiento del personal de apoyo de otras áreas, posibilidad de interacción con otras instituciones). Además, la institución puede influir sobre el desarrollo del proyecto a través de sus políticas internas de promoción a la investigación. En el caso de la Universidad de Antioquia, enmarcada en la denominada Universidad Emprendedora, cuenta para su desarrollo con una unidad de emprendimiento empresarial que le permite fortalecer los proyectos derivados de la investigación universitaria. Morales (2008) define la Universidad Emprendedora como "una universidad con una visión más "empresarial", flexible, con capacidad de adaptación y creatividad para satisfacer las necesidades presentes de la sociedad y para prever el futuro ofreciendo nuevas líneas de estudio, nuevas áreas de investigación y una profunda colaboración con el entorno social y económico".

- Recursos financieros: Este componente se hace necesario para concretar tanto la elaboración como la ejecución del proyecto, ya sea para la adquisición de bienes y servicios como para el pago del personal que hace parte del proceso. En este punto, se podrían obtener recursos financieros tanto del sector público como del privado dependiendo del objeto de investigación y de las gestiones realizadas para la adquisición de dichos recursos, sumado a ello, la forma de obtención de los recursos influye sobre la valoración del proyecto y por consiguiente de la TTU (Correa, 2005).

Grupo de investigación: Se hace necesario conocer el grupo de personas que hacen parte del proyecto, ya que sus conocimientos y experiencia son un factor clave para el éxito del proyecto, además, se debe evaluar la integra-

Elaboración propia.

"La planeación se define como el proceso de llevar la situación presente real a una situación futura deseada, pero posible, que se fija como objetivo. Es así como, las empresas para desarrollar este proceso deben generar planes que materialicen las estrategias y sirvan de guía a la administración para alcanzar los objetivos previamente fijados" (Gutiérrez, 2007, 4). 
ción y consolidación del grupo. Cuando se hace referencia al grupo de investigación, se deben identificar aspectos del capital humano que "se refiere al conocimiento (explícito o tácito), así como a su capacidad para regenerarlo, es decir su capacidad para aprender. Es aquel que pertenece básicamente a las personas, puesto que reside en ellas" (Arango, Pérez \& Gil, 2008).

En la interacción de los componentes de la valoración de TTU, se plantea que los dos componentes de la línea vertical (Grupo de investigación y Proyecto) constituyen los elementos trascendentales para la realización de un proceso de transferencia tecnológica, siendo determinantes, puesto que es en el Grupo donde se gestan los elementos sociológicos que posibilitan la extrapolación de los resultados a la sociedad, esto previa verificación de las potencialidades del Proyecto, su pertinencia y su viabilidad financiera y/o social. Estos elementos los podríamos denominar Componentes Estructurales o Fundamentales (Birley, 2001; Calderón, 2005).

Así mismo, los dos componentes en el eje horizontal (Institución y Recursos financieros), representan elementos, que previo a la existencia de los anteriores, apoyan los proyectos de transferencia. La Institución representa la posibilidad de un respaldo "corporativo" para todo el apoyo logístico, de negociación y de interacción con otras entidades. Los Recursos financieros son un vehículo para la materialización de los proyectos que pueden provenir de diversos agentes tales como entidades estatales, empresas privadas, fondos de capital privado, inversionistas independientes, inversionistas ángeles, entre otros.

Esta combinación de factores permite establecer las relaciones y los canales para la realización de la valoración y la definición de todos los mecanismos que complementan la cadena de valor para la negociación de la tecnología.
Además de lo planteado, se hace necesaria una metodología que permita una aproximación hacia la valoración de los proyectos derivados de proyectos de investigación universitarios. En este sentido, la Ilustración 2, recoge algunos aspectos organizados de forma sistemática que permiten llegar a dicha aproximación, al valor de estos proyectos que cuentan con características especiales, tal como se han expuesto.

Ilustración 2. Metodología para la aproximación a la valoración de TTU ${ }^{4}$

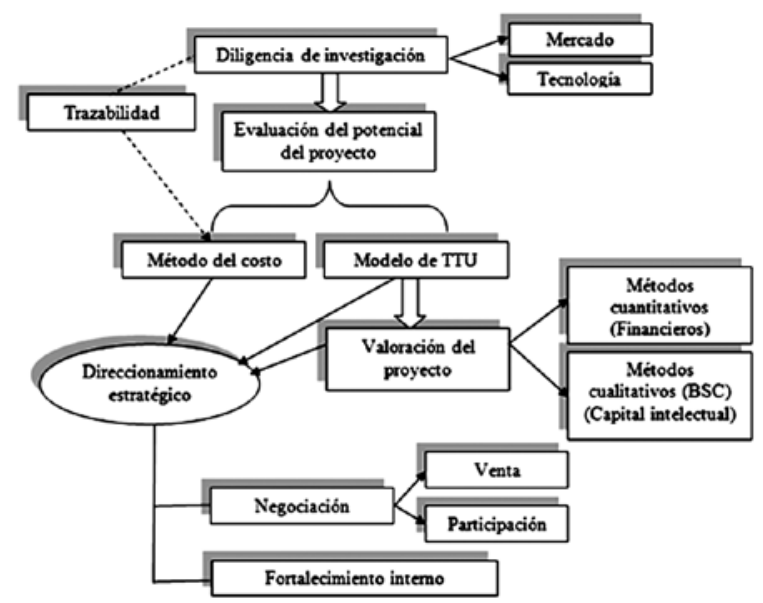

La metodología presentada en la Ilustración 2 establece a nuestro juicio, el conjunto de relaciones que se dan durante el proceso de valoración de una propuesta de transferencia de tecnología con alto valor agregado en una institución universitaria. El planteamiento de manera sintética aborda desde el reconocimiento de la idea empresarial y su potencial hasta la toma de decisiones estratégicas tendientes al direccionamiento estratégico que puede conllevar a alternativas de gestión empresarial diferentes. Este proceso, que constituye una propuesta en el camino de estructurar una metodología de valoración empresarial al interior de las universidades y en particular en la Universidad de Antioquia se describe a continuación:

4 Elaboración propia. 
Diligencia de investigación: Consiste en un conjunto de sesiones con el grupo de investigación en el cual se sensibiliza sobre sus posibilidades de trascender los resultados de investigación mediante la prestación y/o venta de los productos $y / 0$ servicios desarrollados (mejorados). Es un elemento fundamental de esta etapa inicial del proceso, indagar sobre el estado y las condiciones del proyecto en términos del mercado y la tecnología. Sobre el mercado, el interés se centra en evaluar, aunque de manera preliminar, sobre las posibilidades de comercialización que tendría el proyecto, las estrategias que se deben definir para su posicionamiento, las fortalezas y debilidades frente a competidores entre otros aspectos esenciales.

Respecto a la tecnología, se evalúa el grado de maduración de ésta que se transfiere a la sociedad, esto es, si se encuentra en un adecuado punto de desarrollo, o si por el contrario se requiere de más procesos de investigación para consolidar el producto o servicio. En consideración, se hace indispensable una caracterización adecuada de la tecnología desde los aspectos técnicos, una evaluación independiente y objetiva que permita validar los resultados (Osorio et al., 2003).

Los dos elementos antes mencionados, son importantes para decidir con antelación sobre los potenciales que tendría el proyecto derivado de investigación, de no ser así, se requerirá realizar redefiniciones bien sea en el aspecto de mercadeo o de desarrollo tecnológico. Siendo el aspecto de mercadeo el que posee mayores dificultades. Puesto que en ocasiones lo que se pretende comerciar no es tan difundido o no tiene precedentes en el mercado, se hace necesario profundizar en las estrategias comerciales tendientes a la difusión adecuada del producto y/o servicio. Al respecto, la experiencia en el acercamiento a esta tipología de proyectos denota que en algunos casos se trata de mercados focalizados que no requieren de campañas masivas de publicidad, sino de acercamientos puntuales para el conocimiento y venta de los productos y/o servicios. Esta situación se presenta por la alta especialidad, producto del desarrollo científico de los grupos de investigación.
Evaluación del potencial del proyecto: Esta actividad es una conclusión que se alimenta en esencia de la diligencia de investigación, pero que se complementa además con la incorporación de otras variables integrantes del plan de negocios, como son los aspectos técnicos, jurídicos, organizacionales y en algunos casos ambientales y/o sociales. En cada uno de estos aspectos se debe obtener un concepto favorable sobre la viabilidad del proyecto para que éste pueda continuar y avanzar en la definición y consolidación del modelo de negocio y por consiguiente de transferencia de tecnología. Luego de contar con estas definiciones, se tienen elementos esenciales para avanzar en la cuantificación de los costos del proyecto empresarial (metodología del costo) en un concepto de "trazabilidad hacia atrás" o rastreo de la historia del grupo y en particular del proyecto productivo (Vinig \& Van Rijsbergen, 2009).

En este apartado de la viabilidad del proyecto o potencial del proyecto es donde los grupos de investigación requieren de especial ayuda para la estructuración formal de planes de empresa que se conviertan en las herramientas de planeación y de gestión para la puesta en marcha de las spin off universitarias.

Valoración del proyecto: Con este componente de la metodología se pretende dar respuesta a la viabilidad del proyecto en términos financieros con el fin de concluir sobre su posible puesta en marcha y/o búsqueda de potenciales inversionistas. La valoración se realiza a partir de la definición del modelo de TTU y se propone mediante la realización de métodos cuantitativos y cualitativos, los cuales como se anota más adelante, se trata de métodos complementarios.

Aunque la literatura acopiada e incluso las normas internacionales de valoración referencian la metodología del costo como una metodología de valoración, en el presente artículo no se interpreta como tal, debido a que ésta no da cuenta del potencial (en perspectiva) del proyecto $y$ esto es lo que subyace finalmente en una valoración empresarial. 
La relación entre la metodología del costo y las demás seleccionadas para la valoración del proyecto se presenta en que las tres en su conjunto constituyen herramientas para el direccionamiento estratégico del proyecto empresarial, y en particular la metodología enunciada como una herramienta de negociación con la que cuenta el grupo de investigación y más específicamente la Universidad para negociar con base en el proyecto. Con la aplicación de ésta metodología, la Universidad puede argumentar las inversiones realizadas a lo largo de los años de investigación del grupo, para generar los resultados de los que se dispone finalmente para atender y dar respuesta a necesidades de la sociedad (Ortín et al., 2008).

- Direccionamiento estratégico: A este punto ya se dispone de un análisis sobre el potencial del proyecto y su viabilidad de todo tipo, incluyendo el financiero, además de la definición del modelo de TTU centrado específicamente en spin off universitaria y los elementos para la toma de decisiones definitivas del proyecto.

Una de las principales decisiones en el marco estratégico podría ser, el proceso de fortalecimiento interno del grupo de investigación y del proyecto como tal, lo cual en aras del modelo spin off estaría significando un retroceso o un freno en dicho modelo de transferencia, pero que se considera válido en relación con la poca experiencia que se tiene en el país en su aplicación y en los riesgos implícitos en su implementación (COTEC, 2003; Fernández, 2008; Escorsa, Maspons \& Cruz, 2002; Amar et al., 2006).

Otra decisión estratégica, que es concordante con el modelo de TTU, es la Negociación del proyecto empresarial, en la cual el esquema de participación múltiple de inversionistas es el que más responde a la puesta en marcha de spin off universitarias, puesto que en éste concurren varios grupos de inversionistas entre los cuales se incluyen los investigadores, la universidad y los inversionistas privados. El mecanismo de venta del desarrollo empresarial se puede dar principalmente cuando el desarrollo ha sido puntual y de una orientación específica para un sector productivo o una empresa determinada.

Estos elementos descritos en esta metodología recogen lo que a consideración, representa un acercamiento al proceso de valoración de los proyectos de TTU tipo spin off.

\section{Métodos de valoración aplicados}

En esta investigación, se utilizaron los siguientes métodos para la valoración de proyectos de base tecnológica tipo spin off de la Universidad de Antioquia: El método del costo, el método de los flujos de caja descontados y el método de los indicadores.

Los métodos planteados fueron seleccionados porque recogen en sus estructuras los costos necesarios para llegar a los resultados del proyecto (método del costo), además, las expectativas de los flujos de caja futuros donde se identifica la capacidad del proyecto de producir flujos de caja (método de los flujos de caja descontados). Sumado a ello, el método de los indicadores permite recoger aspectos cualitativos que sustentan el valor del proyecto en una posible transferencia tecnológica, facilitando explicar un posible incremento basado en una prima adicional.

Cada uno de los métodos aplicados permite valorar elementos diferentes que aproximan al valor de un proyecto de base tecnológica derivado de investigación, especialmente, para las denominadas spin off universitarias. Desde esta perspectiva, se evidencia mediante indicadores cualitativos que se asumen valores como los recursos invertidos para llegar a la etapa final de proyecto, el valor de los flujos potenciales del proyecto como empresa en marcha, la sustentación del valor y de una posible prima adicional por el alto contenido de valor agregado (International Valuation Standards Council, 2009; Larrán \& Sotomayor, 2005).

Se presentan a continuación cada uno de los métodos aplicados en la metodología para la aproximación a la valoración de la TTU, en el caso aplicado a la Universidad de Antioquia. 


\subsection{El método del costo}

El enfoque del costo, más conocido como el enfoque del costo de reposición depreciado, determina el valor de un activo intangible calculando el costo de reposición de éste por un activo con similar o idéntica capacidad de servicio. Esto establece un máximo para el valor de determinados activos intangibles. Ajustes incluidos con respecto a la amortización, podrían ser requeridos para reflejar diferencias entre el costo de reposición del activo con uno de similar capacidad de servicio (Arango, Pérez \& Gil 2007; Luna \& Solleiro 2007).

Para aplicar el enfoque del costo, el costo de reposición de un activo similar o uno con igual servicio potencial debe ser estimado. Esto debería ser realizado cuando:

- se identifica el precio de reposición del activo en el mercado; o

- se ha determinado el costo de desarrollo o construcción de un activo similar.

En la práctica, pocos tipos de activos intangibles pueden ser estimados de esta forma.

\subsection{Método de los flujos de caja}

Los métodos de los flujos de caja "tratan de determinar el valor de la empresa a través de la estimación de los flujos de dinero - cash flows - que generará en el futuro, para luego descontarlos a una tasa apropiada según el riesgo de dichos flujos" (Fernández, 2008; Hastbacka, 2004). Para esta investigación se utiliza el método de los flujos de caja operativos descontados, utilizando para ello los flujos de caja libre operativos.

En el método de los flujos de caja operativos descontados se hace necesario identificar tres componentes fundamentales de dicho procedimiento: Los flujos de caja operativos, el período de evaluación del proyecto y la tasa de descuento.

- Los flujos de caja operativos (FCLO): Representan la capacidad del negocio de producir flujos de caja desde su operación para cubrir otros pagos no operativos como la deuda y los divi- dendos. La construcción del FCLO parte de la utilidad operacional a la cual se le descuenta el gasto impuesto, dejando como resultado la utilidad operativa después de impuestos (UODI). A la UODI se le suman las partidas que no implican movimiento de efectivo como las provisiones, la depreciación y la amortización, con el fin de obtener el flujo de caja bruto. Luego, se deben sumar o restar las variaciones del capital de trabajo neto operativo (CTNO) y de los activos no corrientes operativos (ANOCO) dependiendo de su impacto sobre los flujos de efectivo de la empresa, obteniendo de esta forma el FCLO.

- El período de evaluación del proyecto: Para realizar las proyecciones del proyecto se debe estimar básicamente el principal período de impacto de los resultados del proyecto de investigación. Para ello se debe tomar como referente el tiempo de obsolescencia de los resultados del mismo, puesto que se trata de productos con alto valor agregado tecnológico que podría ser remplazado en el futuro por un nuevo bien o una innovación.

- La tasa de descuento: Representa la expectativa o demanda de rentabilidad que se le exige al proyecto de inversión. Esta tasa de oportunidad recoge las consideraciones en relación con el riesgo que enfrentan los inversionistas y los distintos agentes de interés en el proyecto empresarial. Para el caso particular de los proyectos de transferencia tecnológica universitaria tipo spin off se debe advertir adecuadamente la tasa de descuento que involucre las expectativas de riesgo del conjunto de inversionistas que puede implicar, tales como los investigadores, la Universidad e inversionistas privados.

\subsection{Método de los indicadores}

El método de los indicadores permite recoger variables cualitativas del proyecto que permiten identificar aspectos que no se incluyen en términos cuantitativos (Bueno, 2008; Dill, 1995). Se pueden analizar los siguientes componentes de forma cualitativa:

- El proyecto: Características, tiempo de ejecución y dedicación y avance tecnológico. Se realiza un análisis DOFA (debilidades, oportunidades, fortalezas y amenazas). 
- El grupo de investigación: Personas que hacen parte del proyecto, estructura del grupo de investigación, objetivos, experiencia en el área del proyecto, resultados obtenidos por el grupo, reconocimiento institucional.

- La institución: Reconocimiento, estrategias de apoyo al emprendimiento, alianzas con el sector público y el sector productivo, apoyo a la investigación, y resultados anteriores de transferencia tecnológica.

Adicional a lo planteado en los anteriores componentes, se puede aplicar la herramienta del Balanced Score Card (BSC) con el fin de determinar metas a cumplir en las diferentes perspectivas que plantea el BSC. Las perspectivas son:

- Perspectiva financiera: El aspecto financiero es al que tradicionalmente se le han diseñado más indicadores. A través de esta perspectiva, se mide si la estrategia institucional está contribuyendo a mejorar la rentabilidad.

- Perspectiva del cliente: Se hace necesario tener claridad sobre los clientes y el mercado en el cual se desarrollan las operaciones.

- Perspectiva de los procesos internos: Se analizan los cambios que deben desarrollarse en los procesos que lleva a cabo la empresa para prestar un servicio de valor agregado para el cliente.

- Perspectiva de aprendizaje y crecimiento: Se medirá la capacidad de la compañía para innovar y adaptarse a las cambiantes condiciones del mercado, debe considerarse el recurso humano y los sistemas de información, la tecnología y el empoderamiento que se entregue de la compañía (Osorio \& Duque, 2003, 62).

Los indicadores a definir por cada perspectiva se construyen dependiendo de las características particulares de la empresa potencial, en la cual se deben definir metas en cada una de las perspectivas.

Los indicadores cualitativos también permiten argumentar el proceso de negociación al momento de transferir la tecnología hacia el sector productivo en una posible venta de los resultados del proyecto de investigación. Además, constituye una guía que aporta conocimiento integral de la organización y sus potencialidades en las distintas áreas (Göktepe, 2005; Hernández, 2006).

\section{Aplicación de la metodología $\mathrm{y}$ análisis de resultados}

Con el fin de acopiar la información cualitativa soporte de esta investigación, se realizaron diversas actividades de trabajo de campo que permitieron la estructuración de la metodología, planteamientos y conclusiones en relación con las variables de investigación y con las premisas que dan lugar a la aplicación de lo propuesto como un punto de partida para la valoración de esta tipología de proyectos en la Universidad de Antioquia y como un mecanismo para hacerlo extensible a otras instituciones.

A continuación se expone el trabajo de campo realizado en relación con las variables de investigación y los componentes del rombo de la valoración de Transferencia Tecnológica Universitaria -TTU- .

- Grupo de investigación:

- Reuniones con el líder del grupo para el direccionamiento del proyecto.

- Acceso a fuentes públicas de información.

- Proyecto empresarial:

- Estudio de la historia del proyecto empresarial establecimiento de la trazabilidad hacia atrás del proyecto CONOSER.

- Análisis del plan de negocio estructurado para el proyecto empresarial.

- Estudio detallado del plan de mercadeo definido para el proyecto.

- Asistencia a presentaciones de proyectos con estas características como jurado.

- Institución universitaria - Universidad de Antioquia:

- Reuniones con los líderes del Programa Gestión Tecnológica -PGT-.

- Sesiones de trabajo aplicadas con el coordinador del concurso.

- Asistencia a reuniones del Comité: UniversidadEmpresa-Estado. 
- Recursos financieros - Fuentes de financiación:

- Sesión de trabajo con el líder de creación de spin off de la Universidad Santiago de Compostela, España; centrando el interés en los mecanismos de financiación para los proyectos de transferencia. Actividad realizada en el marco del Comité Universidad-Empresa-Estado y del programa ERICA.

- Para el caso específico de las fuentes de financiación, consultas a los fondos de capital privado que existen para el financiamiento de este tipo de proyectos.

Una actividad transversal fue la participación activa en el grupo naciente para el estudio de las Spin Off Universitarias que busca consolidar una línea de trabajo en esta temática al interior de la Universidad de Antioquia, de tal forma que se constituya en un apoyo institucional en el liderazgo y consolidación de esta tipología de transferencia de tecnología a la cual la institución le quiere apostar y lo proyecta desde su plan estratégico. El grupo pretende generar la dinámica de estudio e investigación que abandere las temáticas concernientes a las spin off, de tal forma que con base en su trabajo se pueda respaldar a la Universidad en la estructuración de políticas que propendan por la promoción, desarrollo y consolidación de proyectos de transferencia bajo la modalidad Spin Off Universitaria.

\subsection{Análisis componente 1 del Rombo de la valoración de Transferencia Tecnológica Universitaria -TTU-. Grupo de Investigación}

Grupo de Investigación Manejo Eficiente de la Energía -GIMEL- presenta como objetivo principal: Desarrollar investigación y asesoría en el servicio y utilización de la energía eléctrica con miras a una alta competitividad, con consideraciones ambientales y de productividad para el desarrollo sostenible de la región y el país; con el fin de realimentar la formación en ingeniería y otras especialidades en la Universidad de Antioquia.

El grupo de investigación cuenta con las siguientes líneas de investigación bajo las cuales desarrolla sus proyectos sobre ingeniería eléctrica: Compatibilidad electromagnética e impacto ambiental; Gestión de la Energía Eléctrica; Mantenimiento, diagnóstico y protecciones; Mercados Energéticos; Máquinas Térmicas; Robótica y Automatización.

En cuanto a la productividad técnico-científica, se presentan en la tabla 1 los diferentes resultados del grupo de investigación desde su creación en 1996 hasta el 2008, lo que permite evidenciar la gran cantidad de resultados de investigación que han logrado obtener en un período de 12 años.

\subsection{Análisis componente 2 del Rombo de la valoración de Transferencia Tecnológica Universitaria -TTU- Proyecto Empresarial}

El Proyecto de Spin Off Universitaria- CONOSERConocimiento y Servicios de Ingeniería surge del grupo de investigación Manejo Eficiente de la energía (GIMEL) del Departamento de Ingeniería Eléctrica de la Universidad de Antioquia.

CONOSER es una empresa prestadora de servicios de consultoría profesional especializada en sistemas eléctricos que además, ofrece el diseño de equipos para implementar técnicas de mantenimiento predictivo eléctrico en motores de inducción y presta servicios asociados a estos equipos. Actualmente se encuentra en la consolidación de su modelo de negocio, el cual se complementa con el constante desarrollo como grupo de investigación.

Como proyecto de empresa, CONOSER presenta los siguientes productos y servicios que ofrece a sus clientes potenciales: Consultoría y asesoría (Puesta a tierra, Protección contra rayos, Compatibilidad electromagnética, Calidad de la energía); Metodologías y paquetes tecnológicos; Equipos de mantenimiento; Capacitaciones.

A continuación se presenta el análisis $\mathrm{DOFA}^{6}$ del proyecto con el fin de evidenciar los diferentes aspectos que se deben afrontar para lograr el éxito empresarial. Cada uno de los componentes presentados en la tabla 2, responde al análisis del proyecto CONOSER, lo cual se constituye en componentes 
Tabla 1. Producción técnico-científica GIMEL ${ }^{5}$

\begin{tabular}{|l|c|c|}
\hline \multicolumn{1}{|c|}{$\begin{array}{c}\text { Concepto producción } \\
\text { técnico-científica }\end{array}$} & Cantidad & Participación \\
\hline $\begin{array}{l}\text { Artículos publicados en revistas } \\
\text { científicas }\end{array}$ & 120 & $25,37 \%$ \\
\hline Trabajos en eventos & 120 & $25,37 \%$ \\
\hline Libros publicados & 8 & $1,69 \%$ \\
\hline Textos en publicaciones no científicas & 2 & $0,42 \%$ \\
\hline Otra producción bibliográfica & 2 & $0,42 \%$ \\
\hline Software & 17 & $3,59 \%$ \\
\hline Productos tecnológicos & 5 & $1,06 \%$ \\
\hline Procesos o técnicas & 3 & $0,63 \%$ \\
\hline Trabajos técnicos & 14 & $2,96 \%$ \\
\hline Normas & 3 & $0,63 \%$ \\
\hline Cursos de corta duración dictados & 20 & $4,23 \%$ \\
\hline Organización de eventos & 3 & $0,63 \%$ \\
\hline Informes de investigación & 2 & $0,42 \%$ \\
\hline Trabajos dirigidos/tutorías concluidas & 72 & $15,22 \%$ \\
\hline $\begin{array}{l}\text { Jurado/comisiones evaluadoras de } \\
\text { trabajo de grado }\end{array}$ & 7 & $1,48 \%$ \\
\hline Participación en comités de evaluación & 8 & $1,69 \%$ \\
\hline Trabajos dirigidos/tutorías en marcha & 13 & $2,75 \%$ \\
\hline Proyectos & 54 & $11,42 \%$ \\
\hline Total & $\mathbf{4 7 3}$ & \\
\hline
\end{tabular}

esenciales para evaluar su potencial y en una herramienta de gestión determinante a la hora de poner en marcha la empresa.

El potencial que desde lo técnico se evidencia en el proyecto CONOSER se podrá materializar a plenitud, en la medida que se logre consolidar el modelo de transferencia de tecnología que se ha considerado el más conveniente, consistente en una spin off de la Universidad de Antioquia. Esta situación se evidencia, porque el grupo ha venido durante varios años comercializando sus productos y servicios bajo
Tabla 2. Análisis DOFA del proyecto CONOSER ${ }^{6}$

\begin{tabular}{|c|c|}
\hline Debilidades & Oportunidades \\
\hline $\begin{array}{l}\text { Se hace necesaria la creación de un } \\
\text { portafolio de servicios y productos que } \\
\text { incluya los precios. }\end{array}$ & $\begin{array}{l}\text { El valor a pagar por concepto del } \\
\text { servicio relacionado con la inge- } \\
\text { niería eléctrica se puede incre- } \\
\text { mentar gracias a los beneficios } \\
\text { ofrecidos por CONOSER a sus } \\
\text { clientes. }\end{array}$ \\
\hline $\begin{array}{l}\text { Algunos de los servicios prestados por } \\
\text { CONOSER son ofrecidos gratuitamente } \\
\text { por la competencia. }\end{array}$ & $\begin{array}{l}\text { La calidad y el valor agregado } \\
\text { de los productos y servicios de } \\
\text { CONOSER facilitan el acceso al } \\
\text { mercado. }\end{array}$ \\
\hline $\begin{array}{l}\text { El nombre CONOSER no se asocia con } \\
\text { los servicios prestados por el grupo. }\end{array}$ & $\begin{array}{l}\text { El reconocimiento de la Universi- } \\
\text { dad de Antioquia a nivel nacional } \\
\text { le permite a la CONOSER una } \\
\text { mayor acogida en el mercado } \\
\text { potencial. }\end{array}$ \\
\hline & $\begin{array}{l}\text { Los proyectos para racionalizar } \\
\text { el consumo de energía eléctrica } \\
\text { y la búsqueda del desarrollo sos- } \\
\text { tenible. }\end{array}$ \\
\hline Fortalezas & Amenazas \\
\hline $\begin{array}{l}\text { Cuenta con un grupo de profesionales } \\
\text { altamente capacitados y con experien- } \\
\text { cia para prestar servicios en el área de } \\
\text { la ingeniería eléctrica. }\end{array}$ & $\begin{array}{l}\text { Existen gran cantidad de empre- } \\
\text { sas que ofrecen servicios y pro- } \\
\text { ductos eléctricos a bajo costo. }\end{array}$ \\
\hline $\begin{array}{l}\text { CONOSER presenta alto valor agregado } \\
\text { en sus productos y servicios gracias a } \\
\text { que se deriva de un grupo de investiga- } \\
\text { ción de alta calidad. }\end{array}$ & $\begin{array}{l}\text { Algunas de las empresas que } \\
\text { ya se encuentran en el mercado } \\
\text { cuentan con una marca posicio- } \\
\text { nada, especialmente en el área } \\
\text { de las capacitaciones. }\end{array}$ \\
\hline $\begin{array}{l}\text { La investigación hace parte del valor } \\
\text { agregado de la empresa. }\end{array}$ & \\
\hline $\begin{array}{l}\text { Cuenta con apoyo de la universidad de } \\
\text { Antioquia a través del programa "Ges- } \\
\text { tión Tecnológica". }\end{array}$ & \\
\hline $\begin{array}{l}\text { La experiencia de los servicios ya pres- } \\
\text { tados y la satisfacción de los clientes. }\end{array}$ & \\
\hline
\end{tabular}

el amparo del centro de extensión de la Facultad de Ingeniería (CESET - Centro de Servicios Técnicos de la Facultad de Ingeniería).

Fuente: GrupLAC GIMEL, Colciencias (2009).

6 Debilidades, Oportunidades, Fortalezas y Amenazas. Las debilidades y fortalezas responden a factores internos, en tanto que las oportunidades y amenazas a parámetros externos.

7 Fuente: Elaboración propia a partir de la información del plan de negocios del proyecto CONOSER. 


\subsection{Análisis componente 3 del Rombo de la valoración de Transferencia Tecnológica Universitaria-TTU- Institución Universitaria}

Actualmente, la Universidad de Antioquia cuenta con el Programa Gestión Tecnológica (PGT) el cual hace parte de la Vicerrectoría de Extensión. A través del PGT y de sus unidades de emprendimiento empresarial y transferencia de tecnología, la Universidad ha podido apoyar a los grupos de investigación universitarios en los procesos de diseño de planes de negocio y creación de empresas formalizadas, lo que demuestra el interés de la institución para colaborar a los estudiantes, docentes y comunidad en general en sus actividades de emprendimiento empresarial y a los grupos de investigación internos a consolidar sus proyectos para realizar procesos de transferencia.

Las relaciones que se desarrollan con la comunidad, especialmente con el sector empresarial, han facilitado la relación Universidad-Empresa-Estado, puesto que la Universidad administra recursos públicos que son direccionados hacia actividades de investigación y extensión que buscan finalmente impactar en las empresas, bien sea a través de la creación de nuevos diseños tecnológicos, técnicas de aplicación empresarial, metodologías para la optimización de recursos, o por medio de la creación de nuevas empresas denominadas en el ambiente universitario como Spin Off, las cuales contienen un alto valor agregado de $\mathrm{I}+\mathrm{D}$ y que generan nuevos empleos directos $e$ indirectos. Además, se debe tener en cuenta que la Universidad se encuentra en línea con las políticas de investigación y desarrollo promovidas por el Estado colombiano.

Año tras año se vienen incrementando los proyectos $\mathrm{de} \mathrm{I}+\mathrm{D}$ relacionados directamente con el sector empresarial propiciando con ello el acercamiento de la Universidad con los sectores social y productivo.

En materia de generación de recursos, la Universidad de Antioquia cuenta con elementos normativos vigentes y en proceso de elaboración tendientes a estructurar y regular las relaciones en materia de transferencia de tecnología y específicamente para el caso de las spin off, lo cual la hace una universidad líder en el tema a nivel regional y nacional, pese al atraso que en esta materia se evidencia en universidades de talla internacional. Estos instrumentos son vitales porque son herramientas que denotan procesos de formalización en la gestión tecnológica.

La tabla 3 pone de manifiesto el incremento paulatino de la investigación mediante los fondos universitarios, lo que posteriormente ha de redundar en resultados de investigación escalables y con la posibilidad en el mejor de los casos, de consolidarse como empresas autónomas.

\subsection{Análisis componente 4 del Rombo de la va- loración de Transferencia Tecnológica Uni- versitaria-TTU- Recursos financieros}

El proyecto CONOSER visto como Spin Off Universitaria, se debe considerar en términos financieros como una empresa autónoma con las particularidades que le imprime el hecho de su gestación al interior de una institución universitaria. En este orden de ideas se distinguen las siguientes fuentes de financiación (total y/o parcial) para la puesta en marcha y operación como empresa:

- Financiación autónoma, Generación interna de fondos: Corresponde a los recursos que puede generar el proyecto empresarial en desarrollo de su actividad misional. En el largo plazo y en el período de evaluación financiera del proyecto, esta fuente de financiación debe primar sobre las otras, porque es la que garantiza que éste es sostenible bajo el modelo de negocio y de transferencia de tecnología seleccionado.

Se deben considerar los recursos que haya generado el grupo de investigación hasta la fecha de puesta en marcha del proyecto como empresa, pues estos recursos se podrían aportar como parte de la inversión inicial del proyecto y aumentar la participación de los investigadores en los resultados de la explotación de la spin off.

- Facultad, centro de extensión y de investigación: Las unidades académicas de la Universidad de Antioquia (Facultades, Escuelas, Corporaciones e Institutos) cuentan con recursos de discrecio- 
Tabla 3. Comportamiento de la inversión en los fondos de apoyo a la investigación: 2002 al $2008^{8}$

\begin{tabular}{|c|c|c|c|c|c|c|c|}
\hline FONDO & 2002 & 2003 & 2004 & 2005 & 2006 & 2007 & 2008 \\
\hline Regionalización & & & $35,000,000$ & $23,881,000$ & & $50,000,000$ & $26,182,500$ \\
\hline Pequeños proyectos & & & & $109,208,200$ & & $130,000,000$ & $39,272,000$ \\
\hline Trabajos de Grado & $7,200,000$ & $8,388,900$ & $16,136,500$ & $17,995,000$ & $14,395,675$ & $20,000,000$ & $27,830,350$ \\
\hline Jóvenes Investigadores & $180,641,684$ & $290,366,300$ & & $276,172,648$ & $181,166,666$ & $202,000,000$ & $200,000,000$ \\
\hline Pasajes Internacionales & $82,310,612$ & $91,363,936$ & $121,304,997$ & $130,913,051$ & $111,134,918$ & $160,000,000$ & $193,065,687$ \\
\hline Pasajes Nacionales & $9,880,000$ & $9,149,211$ & $3,539,500$ & $5,279,920$ & $4,930,000$ & $8,000,000$ & $13,056,383$ \\
\hline $\begin{array}{l}\text { Investigadores de } \\
\text { Trayectoria }\end{array}$ & $27,088,628$ & $29,837,926$ & $41,174,042$ & $31,281,874$ & $45,049,721$ & $70,000,000$ & $53,059,797$ \\
\hline Reparación Urgente & $18,441,178$ & $4,056,800$ & $6,106,676$ & $24,060,736$ & $17,390,211$ & $30,000,000$ & $26,138,240$ \\
\hline Revistas & $33,628,750$ & $35,000,000$ & $39,580,000$ & $33,400,000$ & $38,398,500$ & $45,000,000$ & $26,568,000$ \\
\hline Revistas Indexadas & & & & & $28,478,458$ & $82,887,082$ & $122,767,880$ \\
\hline Eventos & $26,283,841$ & $21,000,000$ & $25,325,000$ & $32,234,000$ & $29,148,000$ & $40,000,000$ & $37,000,000$ \\
\hline Bioética & 920 & 500 & 941,92 & $6,079,892$ & $1,349,800$ & $5,000,000$ & \\
\hline $\begin{array}{l}\text { Contrapartidas e } \\
\text { Incrementales }\end{array}$ & $470,100,139$ & $443,632,890$ & $430,133,616$ & $335,930,779$ & $542,705,057$ & $584,231,717$ & $684,695,758$ \\
\hline Evaluaciones Externas & $37,929,373$ & $28,303,159$ & $36,013,685$ & $35,254,000$ & $6,465,000$ & $74,789,061$ & $47,385,000$ \\
\hline $\begin{array}{l}\text { Investigación Aplicada, } \\
\text { Innovación y propiedad } \\
\text { intelectual }\end{array}$ & $1,214,000$ & $122,987,236$ & & & $180,000,000$ & & \\
\hline \multicolumn{8}{|l|}{ Capacitación } \\
\hline Jornadas de Investigación & & & & $14,428,992$ & $14,811,504$ & & $31,020,865$ \\
\hline Total & $895,638,205$ & $1,084,586,358$ & $755,255,936$ & $1,076,120,092$ & $1,215,423,510$ & $1,501,907,860$ & $1,528,042,460$ \\
\hline
\end{tabular}

nalidad producto de sus actividades de investigación y extensión. Estos recursos podrían ser destinados por la unidad académica a aportar a la financiación inicial de las demandas de inversión que requiere el proyecto empresarial; de esta manera la participación de la Universidad aumentaría en la spin off, ya que la Facultad no cuenta con independencia jurídica para figurar como tal. Los beneficios que recibiría la unidad académica se canalizarían por la administración central de la Universidad y la distribución de beneficios se haría de acuerdo a las normas que internamente se definan para tal efecto. No obstante los recursos autónomos disponibles de cada Facultad se pueden destinar en las etapas iniciales del proyecto empresarial a estimular y ayudar a consolidar el modelo de negocio que se gesta para la transferencia de tecnología.

- Universidad, fondo de innovación y demás fondos patrimoniales: Hace referencia a los recursos de la administración central que se orientan al fomento de proyectos con alto valor agregado y que pueden constituirse en spin off. El fondo de innovación de la Universidad de Antioquia, tiene desde su concepción el apoyo a distin-

8 Fuente: Universidad de Antioquia (2008). 
tas actividades de los grupos de investigación tendientes al establecimiento de proyectos que trascienden las actividades habituales realizadas al interior de la Universidad. Igualmente y aunque aún no está reglamentado, la Universidad podría destinar recursos de sus fondos patrimoniales especiales a apostar a la creación de empresas gestadas desde la Universidad. Un ejemplo de mecanismos alternativos de financiación.

- Convenios interinstitucionales: El sector privado y el sector público cada vez ha tomado mayor conciencia de la importancia de la Universidad y su potencial de investigación como un aliado estratégico en su cadena de valor, pues cuenta con toda la infraestructura investigativa y organizacional para generar soluciones efectivas a sus problemáticas específicas. En este orden de ideas, una estrategia de financiación de spin off universitarias es la realización de alianzas y/o convenios interinstitucionales con distintas entidades, de tal forma que de entrada se estipule que los resultados obtenidos de los procesos de investigación darán lugar a una unidad empresarial independiente de la Universidad con la participación accionaria del ente financiador. En muchas ocasiones la entidad que financia los procesos de investigación lo hace sólo a manera de investigación contratada para una aplicación puntual sin el interés de generar una empresa independiente, no obstante aunque este último mecanismo no se acomode al esquema spin off si constituye un actor determinante para la financiación de la investigación y aplicar otros modelos de transferencia de tecnología universitaria.

- Fondos de capital privado (de riesgo): Estos mecanismos de financiación de proyectos empresariales y de impulso a empresas existentes constituye un modelo de cierta manera reciente en Colombia. Es un esquema de financiación que propende por impulsar proyectos o empresas jóvenes con gran potencial. El otro componente se denomina emisores por primera vez y está orientado a promover que más empresas ingresen al mercado público de valores como emisores de títulos. El esquema de los fondos de inversión privado se adapta en gran medida a las necesi- dades que demandan los proyectos tipo spin off, no obstante hay cierta reticencia por parte de los inversionistas a realizar alianzas con las universidades y a depositar sus recursos en proyectos empresariales que generalmente son de un riesgo alto por responder a tecnologías novedosas que no siempre han sido probadas con antelación. Esta alternativa de inversión es ampliamente usada en otras latitudes para la financiación de proyectos de spin off. Finalmente, se puede decir que las principales debilidades de este mecanismo de inversión en relación con la modalidad de proyecto de transferencia que se plantea, radica en los montos mínimos de inversión que ellos apoyan y que no todos los fondos están en disponibilidad de invertir en proyectos universitarios por la complejidad en el manejo de las relaciones en el marco del gobierno corporativo.

- Inversionistas privados: Hace referencia a la intencionalidad individual que puedan manifestar inversionistas por depositar sus recursos en esta tipología de proyectos. Aunque no es el mecanismo de financiación más ortodoxo para la dinámica de estos proyectos, es una alternativa a considerar para algunos casos particulares.

\subsection{Resultados de las metodologías de valoración aplicadas}

La aplicación y desarrollo de herramientas gerenciales con grupos de investigación es una labor ardua tanto para quien adelanta el trabajo de consultoría como para los integrantes del grupo, pues estos, en la mayoría de los casos no gozan de una experticia para atender a los requerimientos del consultor y éste a su vez, no domina los conceptos altamente técnicos que envuelven el proyecto empresarial objeto de análisis. No obstante, superadas estas dificultades y con la solución de estas asimetrías, el proceso para la aplicación de las metodologías de valoración se torna bastante interesante, pues esa interacción entre profesionales técnicamente formados en un campo del conocimiento y el consultor con su formación mucho más empresarial, redunda en beneficios para el proyecto y para la institución universitaria que se alimenta de experiencias para fortalecer los demás procesos de creación de spin off universitarias. 


\section{Consideraciones finales}

Las evidencias teóricas no dan cuenta de una amplia difusión de modelos de valoración de proyectos de transferencia tecnológica aplicados por cada universidad. Lo que se encuentra son experiencias de distintas universidades que han realizado procesos de transferencia con el sector privado o con el Estado. Esta situación lleva a que se deba estructurar una agrupación de temáticas que combinadas logren dar respuesta a la problemática objeto de estudio.

Los resultados permiten una plataforma de negociación, puesto que se logra una aproximación al valor de los proyectos de base tecnológica que cuentan con altos contenidos de valor agregado y de capital intelectual y facilitan el acompañamiento de la universidad no sólo en aspectos técnicos y administrativos, sino también en el área financiera.

En términos generales, el trabajo desarrollado busca sentar bases para la estructuración y formalización de metodologías más consistentes al futuro, de tal forma que cuando los grupos de investigación produzcan resultados susceptibles de ser transferidos cuenten con un soporte institucional en el proceso de valoración.

\subsection{Respecto al Grupo de investigación}

Un aspecto que puede dificultar en un momento dado los procesos de transferencia mediante el modelo spin off, es la falta de disponibilidad de los integrantes del grupo de investigación a entrar en un plan de emprendimiento empresarial, esto es, porque algunos carecen de visión como empresarios y su visión es meramente académica. Esta situación se ve superada cuando al grupo emprendedor universitario se integran personas con perfil gerencial y empresarial, que si bien no han participado en los desarrollos técnicos del grupo si pueden potenciar sus resultados en el mercado. Por tanto se hace pertinente que las unidades (oficinas) de transferencia de tecnología acompañen a los grupos de investigación en la consolidación de su modelo empresarial, de esta forma se permite aprovechar todo el talento humano que se puede obtener en una universidad mediante la realización de alianzas con otras unidades académicas que puedan aportar el recurso humano necesario para dichas gestiones y que se conserve la visión universitaria del proyecto.

La alta movilidad (rotación) que puedan tener los integrantes del grupo de investigación se puede constituir en un factor que determine en gran medida los resultados de los proyectos de investigación. No obstante, si los líderes de las líneas de investigación permanecen en el tiempo y estos a su vez, hacen escuela incorporando estudiantes en formación que vayan alimentando y reforzando el trabajo de base del grupo, se puede pensar en la continuidad de los procesos.

\subsection{Respecto al Proyecto Empresarial}

El proyecto al margen de provenir de una universidad sin importar si ésta es pública o privada, se debe evaluar bajo el amparo de políticas y parámetros de un proyecto externo, esto es, como un proyecto empresarial privado con la particularidad puntual de ser un proyecto innovador.

Se debe tener consideración en relación con la vida útil del proyecto y la capacidad de renovación y/o actualización que se tiene por parte del grupo de investigación (o del proyecto) que lo respalda. Esta variable es determinante a la hora de hacer la evaluación, puesto que es una de las premisas claves en la evaluación mediante los métodos de flujos de caja y es analizado también por los inversionistas para la estimación de posibles inversiones que permitan garantizar que los proyectos empresariales estén a la vanguardia de la tecnología (dura y blanda) y en consecuencia puedan permanecer en el tiempo de manera sostenible.

\subsection{Respecto a la Institución Universitaria}

La Universidad de Antioquia ha realizado procesos de transferencia tecnológica y trabaja actualmente para aumentar la oferta al sector público; pero hasta ahora las negociaciones no se han soportado en modelos formalmente estructurados. Desde la Vicerrectoría de Investigación se ha manifestado expresamente la necesidad de estructurar un modelo de valoración que soporte los procesos de transferencia tecnológica. 
La falta de incentivos económicos y de otro tipo para que más grupos de investigación se involucren en la dinámica de generación de proyectos productivos con soluciones reales a la sociedad, se ha identificado como una de las mayores limitantes a la hora de pensar en la incursión en modelos de transferencia de tecnología complejos, tales como las spin off universitarias.

Estructuras y regulación específica que legisle sobre los derechos de propiedad de la institución, en particular aspectos como el uso de la marca, alcances en la participación de la Universidad en las Spin Off, relaciones posteriores del grupo de investigación con la empresa, con los estudiantes y en general con la estructura académica de que dispone la universidad; son factores vitales para que esta modalidad de transferencia de tecnología se pueda instaurar como un modelo efectivo en las universidades colombianas. En el caso particular de la Universidad de Antioquia, en la actualidad se cuenta con un grupo naciente de estudio en el tema de spin off acompañado desde el Programa Gestión Tecnológica, que busca ser el soporte y promotor de estas iniciativas que conlleven a la concreción de los resultados de investigación en modelos de transferencia realmente exitosos al servicio de la sociedad colombiana.

\subsection{Respecto a las Fuentes de recursos financieros}

Las estrategias regionales y nacionales para afianzar los lazos entre la Universidad, las Empresas y el Estado se han ido constituyendo en canales para el mejoramiento de las relaciones entre estos actores. Dicha situación ha permitido que tanto los empresarios privados como el Estado a distintos niveles, vayan volcando sus intereses hacia la universidad $y$ sus fortalezas en investigación. De esta manera se han empezado a realizar alianzas estratégicas para la realización de proyectos de investigación conjuntos que buscan atender las problemáticas sociales que demandan acciones y esfuerzos conjuntos. Ejemplos recientes lo representan las alianzas promovidas por el Ministerio de Agricultura para la realización de varios trabajos de investigación con universidades del país, la creación de centros de excelencia con apoyo y participación de la empresa privada y el reciente acuerdo de cooperación entre la Fábrica de Licores de Antioquia y la Universidad de Antioquia para la investigación de nuevos productos y mejoramiento de los actuales, de tal forma que la citada empresa pueda ser más competitiva en el tiempo.

La situación descrita ha permitido que los inversionistas consideren la Universidad como un aliado potencial y un destino de sus recursos; situación que sin duda abre las puertas a un mayor nivel de desarrollo para los grupos de investigación y por supuesto para la universidad a la cual pertenecen. Este cambio paradigmático se alinea también con perfiles de inversionistas con baja aversión al riesgo, especialmente cuando invierten en proyectos de investigación que se encuentran en sus etapas exploratorias puesto que hay mayor nivel de incertidumbre sobre los posibles resultados del proyecto.

La creación de fondos de inversión especializados que se orienten al potenciamiento de proyectos productivos con alto valor agregado provenientes de las universidades, constituye sin lugar a dudas un avance significativo en este sentido, pues abre las puertas a este grupo de potenciales empresarios de contar con aliados para el financiamiento de los proyectos productivos. No obstante es importante que estos fondos se interesen por financiar proyectos a distintos niveles de requerimientos de capital, ya que a veces sólo financian proyectos muy cuantiosos y no todos tienen los proyectos tipo spin off o tienen las dimensiones y requerimientos mínimos de inversión que estos exigen. Se considera en consecuencia que los filtros para el otorgamiento de capital deben responder más a las especificaciones técnicas del proyecto, el capital humano y la institución universitaria que lo respalda, más que el monto requerido de inversión.

\subsection{Respecto a la aplicación de las metodologías de valoración}

Las diversas metodologías existentes para la valoración de proyectos permiten evidenciar la necesidad de diseñar nuevas propuestas en torno a la valoración de proyectos de base tecnológica que recojan aspectos que no se han tenido en cuenta hasta el momento, especialmente, en las universidades que 
buscan mejorar su impacto en la sociedad a través de las spin off que surgen de los proyectos de investigación que se emprenden desde allí.

La valoración de proyectos de base tecnológica desarrollados a partir de la investigación universitaria se constituye en un aspecto de gran relevancia no sólo para la Universidad de Antioquia, sino para las diferentes instituciones a nivel nacional $e$ internacional que requieren de modelos y metodologías que permitan lograr un acercamiento hacia la medición de los resultados de sus procesos investigativos. Además, se hace necesario transferir los desarrollos logrados por la academia hacia la sociedad en general y las empresas en particular, para lo cual es indispensable contar con una base de negociación que facilite el intercambio y permita que las universidades generen ingresos para continuar con la faceta investigativa que las caracteriza.

Metodologías de valoración más sofisticadas como la de opciones, se pueden comentar como metodologías de vanguardia y firmes candidatas a ser consideradas en la valoración de proyectos con las características de las spin off universitarias. No obstante, esta clase de metodologías aún no son muy difundidas en la comunidad empresarial y no gozan de alta practicidad para su fácil aplicación. Es por esto, por su complejidad y por la inexistencia de metodologías anteriores aplicadas en la Universidad de Antioquia que se optó por las tres metodologías aplicadas pues se consideran adecuadas, pertinentes, accesibles en su aplicación y que responden de manera efectiva a los requerimientos de la Universidad en esta materia.

\section{Referencias}

Amar, P., Vega, J., Ortega, M. \& Quintero, J. (2006) La relación universidad empresa como estrategia del estado para el fomento a la innovación. En: X Congreso Internacional de Ingeniería de Proyectos. Valencia, 13 a 15 de septiembre. España.

Arango, M., Pérez, G. \& Gil, H. (2008) Propuestas de modelos de gestión de capital intelectual: una revisión. En: Contaduría Universidad de Antioquia, 52: 105-130.

Arango, M., Pérez, G. \& Gil, H. (2007) Aspectos prácticos de la gestión del conocimiento y la innovación aplicada a las empresas. Medellín: Editorial Universidad Nacional de Colombia Sede Medellín.
Birley, S. (2001) Universities, Academics, and Spinout Companies: Iessons from Imperial. En: International Journal of Entrepreneurship Education, 1(1): 133-154.

Bueno, E. (2008) La tercera misión de la universidad: el reto de la transferencia del conocimiento. Universidad, empresa - Estado, 1, enero - junio, 76-80.

Calderón, M. (2005) Políticas de transferencia tecnológica para la atracción de recursos en las universidades. En: Revista de derecho y tecnologías de la información, 3: 1-15.

Correa, J. A. (2005) De la partida doble al análisis financiero. En: Contaduría Universidad de Antioquia, 46: 169-194.

COTEC. (2003) Nuevos mecanismos de transferencia tecnológica. Madrid: Fundación COTEC para la innovación tecnológica.

Dill, D. (1995) University-industry entrepreneurship: The organization and management of American university technology transfer units. En: Higher education, 29: 369-384.

Escorsa, P., Maspons, R. \& Cruz, E. (2002) Inteligencia competitiva y transferencia de tecnologías: reflexiones para el desarrollo de la relación universidad-empresa (pp. 1-16).

Fernández, P. (2008) Métodos de valoración de empresas. Documento de investigación DI-771. IESE business school, Universidad de Navarra.

Göktepe, D. (2005) Investigation of university industry technology transfer cases: a conceptual and methodological approach. Working paper (pp. 1-38).

Gutiérrez, J. (2007) Modelos financieros con Excel. Bogotá: Ecoe Ediciones.

Hastbacka, M. (2004) Technolgy valuation - The market comparables methods. En: Technology management journal, june, 1-4.

Hernández, C. (2006) Knowledge transfer opportunities for the bioscience sector in Chile. En: Journal of technology management and innovation, 3(1): 4-16.

International Valuation Standards Council. (2009) Revised International Valuation Guidance Note No. 4. Valuation of Intangible Assets.

Larrán, J. M. \& Sotomayo, S. (2005) Valoración y reconocimiento de activos intangibles. En: Revista Internacional Legis de Contabilidad y Auditoría, 21, Enero - Marzo: 83-128.

Luna, K. \& Solleiro, J. (2007) La gestión de la propiedad intelectual en centros de investigación mexicanos: el caso del instituto mexicano de petróleo. En: Journal of technology management \& innovation, 2(2): 157-169.

Morales, S. T. (2008) El emprendedor académico y la decisión de crear spin off: un análisis del caso español. Tesis doctoral, Departamento de dirección de empresas, Universidad de Valencia, Valencia, España.

Ortín, P, Salas, V., Trujillo, M. V. \& Vendrell, F. (2008) La creación de spin off universitarias en España: características determinantes y resultados. En: Economía industrial, 368: 79-95.

Osorio, J. A. \& Duque, M. I. (2003) Modelos de medición y desempeño interrelacionados en la contabilidad de gestión. En: Tecnología administrativa, 38(XVI): 45-68.

Universidad de Antioquia (2008) Documento Balance Social 2008 Universidad de Antioquia. Vicerrectoría de Investigación - Universidad de Antioquia.

Vinig, T. \& Van Rijsbergen, P. (2009) Determinants of university technology transfer: comparative study of US, Europe and Australian universities. Working paper (pp. 1-25). 